\title{
Analysis based on Bayesian Model of the Spatial Variation of Hospitalization Admissions for Hypertension Disease in Karachi, Pakistan
}

\author{
Madiha Khurram Pasha \\ Department of Computer Science \\ University of Karachi, Karachi, Pakistan
}

\author{
Sidrah Yousuf \\ Department of Computer Science \\ University of Karachi, Karachi, Pakistan
}

\begin{abstract}
Hypertension, also known as Arterial Hypertension (HTN), is a long term and constant disease in which pressure of blood in the arteries is higher than the normal condition of its flow. Normally Blood pressure is measured by two levels i.e. Contraction (Systole) and relaxation (Diastole), these two are the maximum (Systolic) and minimum (Diastolic) blood pressure conditions. Hypertension can defaced the body organs and also lead many other illnesses and diseases like kidney failure, Heart attack and Heart failure etc. in middle age high rate of blood pressure may cause the severe decline in during the whole life time. For the purpose to calculate the spatial variation; to measure specific quantity at varies locations with the help of Descriptive Statistics by using Bayesian Model which reveals and identify some unique values and range of hospitalization admission for hypertension disease across Karachi city of Pakistan. This study of spatial variation of hospitalization admissions for hypertension disease is basically a framework which being developed for the specific purpose to control the high risk of hospital admissions in certain areas and aware about its varies rise and fall in the specific regions as well as provide useful information for concerned authorities and administrators in health department for its betterment and implementation to enhance the ease for hospital admission and reduce the rate of hypertension in certain age group. After this research further modification in the related title should explain the spatial patterns and in this regard should have some detailed and vast collection of Hypertension disease hospitalization records and data.[2][6]
\end{abstract}

GENERAL TERMS: Hypertension, spatial variation, Bayesian model, Rheumatic, congenital.

KEYWORDS: Arterial hypertension (HTN), Bayesian Model.

\section{1- INTRODUCTION}

WHO (World Health Organization) showed that Hypertension as a long standing in most common diseases of world and it is a basic cause of mortality due to cardiac attack and mortality of cerebellum or cerebral vascular mortality. Hypertension itself major part of heart failure, arterial disorders, strokes and many other long term or chronic diseases. The cure and its relative treatment for the critical diseases caused by Hypertension lead high and costly medical resources to cure those kinds of diseases.

$\$ 91.4$ billion cost has been estimated in 2015 for the treatment of high BP by the Heart Association of America. [2][4]
Cardiovascular diseases worldwide have high range of risk approximately over $30 \%-40 \%$ in contrast of other disease. Hypertension disease is main cause or high rated cause of death. According to WHO Hypertension disease was the major cause of approximately 3,512,000 deaths in developed countries and in developing and under privileged countries the value is almost 2,484,000 mortalities by Hypertension disease.

In Pakistan, the Hypertension disease has no age, total of $1 \%$ of all infants born in this country have hereditary Heart disease, about $6 \%$ school going children have Heart disease Rheumatic type and mainly $15 \%$ population of Pakistan suffers from Hypertension disease (High B.P) and especially by Angina and Heart Attacks. Most of the Cardiac patients lose their lives within first inception of symptom in almost one hour because they admitted very late to hospital. The death rate is approximately $90 \%$ due to ventricular fibrillation preceding sudden Heart attack. [4][7]

In Pakistan, the rate for treatment and mediation for hypertension diseases is very low in comparison with well developed countries. In urban regions of Karachi city the population and aging rate is also high and it is increasing day by day. There are very limited studies have been attempted by researchers and other concerned authorities regarding spatial variation on Hypertension disease over Urban Regions of Pakistan, Even epidemiological studies not fully describe the geo-referenced data about Hypertension disease. In this paper we have implement and applied Bayesian (Hierarchical) Models to explain and discover the spatial variation of relative threat for hospitalization admissions of Hypertension disease throughout Karachi, Pakistan. One indirect factor is "Road Density" which is used in Bayesian models as a covariate on relative threat. The final model contain featured obstruct and spatial constituent. These constituents of spatial and intercept modeling is Structured and in some way it is Unstructured. [7][12]

Spatial analysis utilized to visible the pattern of Hypertension disease admissions and also scanned statistics of spatial variation of the standard data in Bayesian modeling also map the bunch or clusters. The outcomes identified that the relative threat for hospital admission for Hypertension disease has clusters of large value in low socioeconomic areas. This research deals with some of the highly relative risky regions which are facing the hospitalization admission issue for Hypertension disease. [13]

The Data for this purpose is collected from many sources and commonly known hospitals of Karachi city especially from lower socioeconomics regions are included with their facts and figures conducted from recent records of years 2013 to 2014.The data is also tabulated with all relative features 
including the levels High and Low in particular sub-districts with "Standardized ratio calculation" and "Bayesian model". [12]

\section{2- METHODS AND MATERIALS}

\subsection{Description of the Study Area}

Karachi is situated in one of the province of Pakistan i.e. SINDH, located on the Coastline of Arabian Sea. It is also known as "Capital of Sindh" as well as "Gate way of Pakistan".

It is the most populous city and center of Pakistan's Banking, finance, economic and industries etc. It is the main seaport of Pakistan. It is popular due to its night life that's why known as "City of Lights" (i.e. the city which never sleeps). This Metropolitan city has an about 23.8 million population estimated by 2013 and the area is approximately $3,527 \mathrm{~km}$ 2.the density of people is approximately 6,000 per square Kilometer. [1] (See Figure 1, 2 and 3).

Karachi has also ranked 2nd largest city amongst all the cities of world regarding population and ranked 7 th as the largest Urban Cluster in the world.

Karachi is an also the biggest center for Medical research and many other academic and developmental studies and their most of other aspects as well as the biggest knowledge hub in Asia and in most of the other Muslim countries.

As it's a big metropolitan populous city that's why it also contain high risk of Hypertension disease in many areas and hospitalization admission problems are also faced by many locals especially in lower socioeconomics regions of Karachi.[1] (see Figure 3).

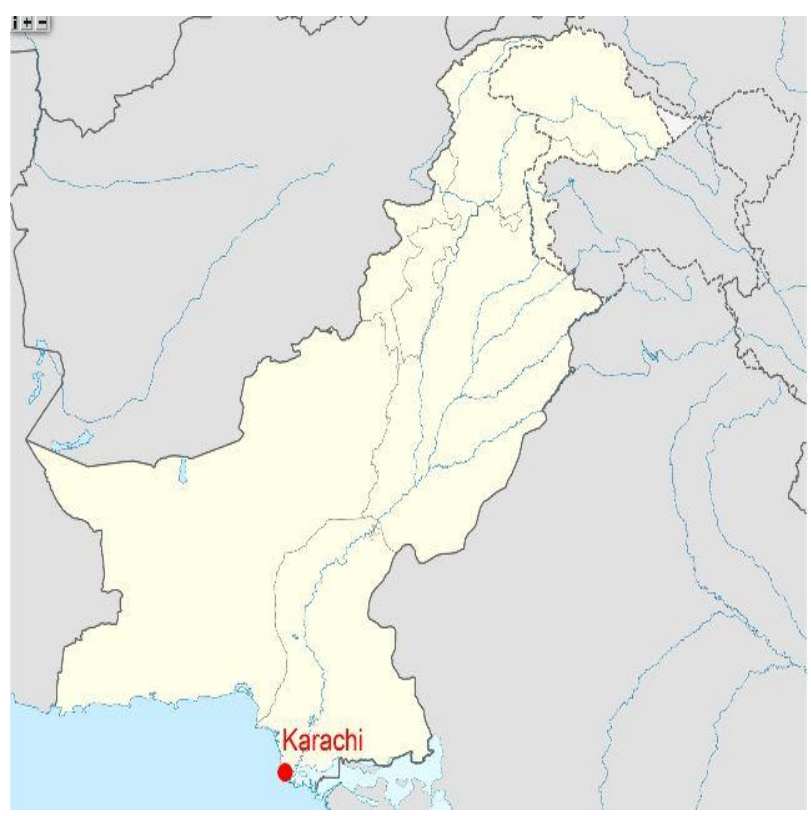

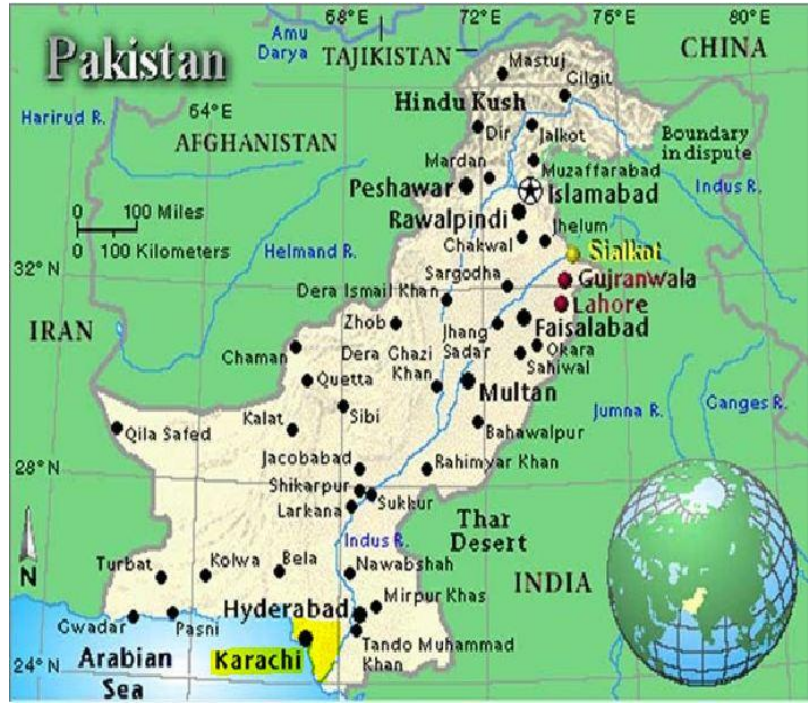

Figure 2: A Map of Pakistan showing the location of Karachi [1]

www.en.wikipedia.org/wiki/Karachi.Information about Karachi City. Retrieved 6 January 2014

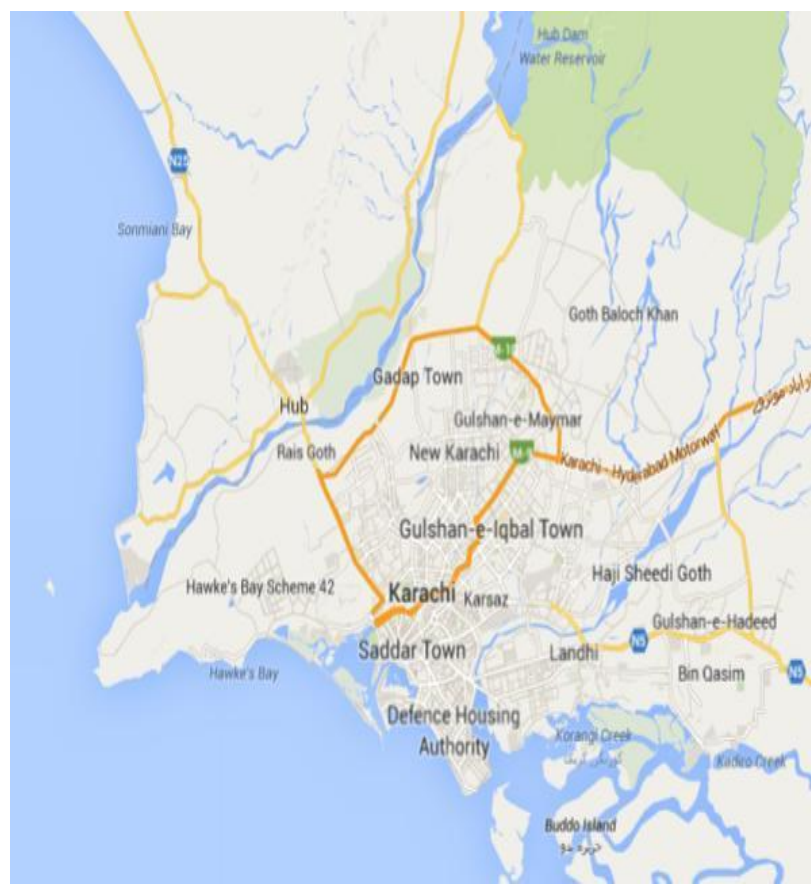

Figure 3: A Map of Karachi [1]

www.en.wikipedia.org/wiki/Karachi.Information about Karachi City. Retrieved 6 January 2014

Figure 1: Map of Pakistan showing the location of Karachi [1]

www.en.wikipedia.org/wiki/Karachi.Information about Karachi City. Retrieved 6 January 2014 


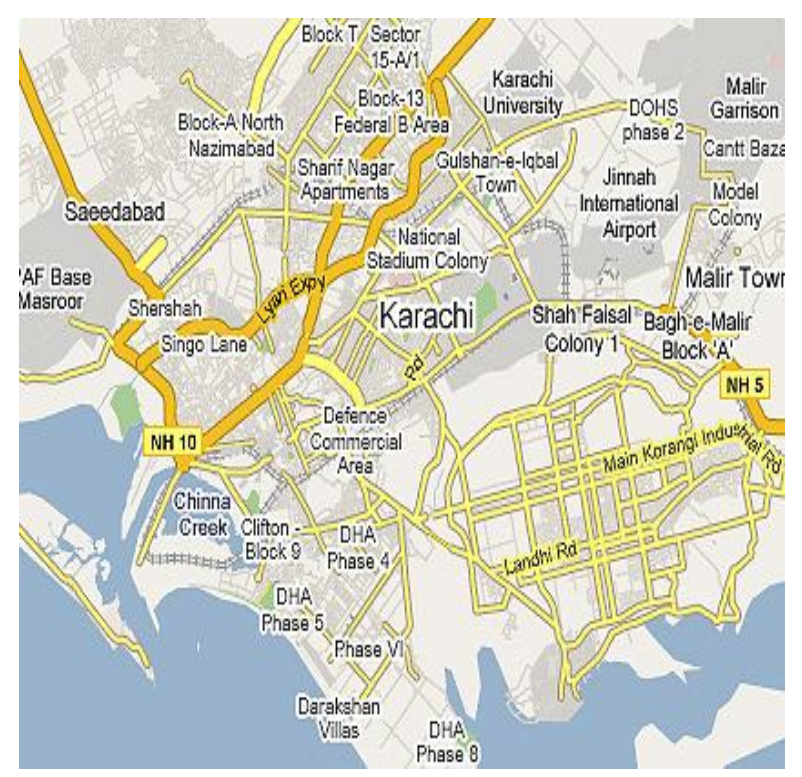

Figure 4: Map of Karachi [1]

www.en.wikipedia.org/wiki/Karachi.Information about Karachi City. Retrieved 6 January 2014

\subsection{Data}

In Karachi, the rate of hypertension disease is continuously increasing since past 60 years. According to other medical research on Hypertension disease that the frequency of hypertension diseases increasing day by day mostly in low socioeconomic regions of the Karachi city. [2] (See Figure 5). [3]

The describing data in this paper mostly contained the original data of many hospitals of Karachi city especially low socioeconomic areas. This data is collected on the basis of known frequency risk factors of Hypertension disease hospitalization admissions. According to the collected data from one of the Hospital located in "Orangi Town" named as "Sindh Govt. Qatar Hospital, Karachi", from past 10 years, the patient above the age of 28 with major hypertension risk various modified factors for Hypertension and its major outcome is noted at the time of discharge from Hospital. The collected data shows the approximate information about the factors that is approximately seventy five percent (75.4\%) out of ninety five $(95 \%)$ patients had hypertension disease and severe cardiac attack /stroke. Almost sixty (60\%) percent hypertension modified risk factor followed by heavy smoking habit forty five (45\%), with Diabetes in twenty five (25\%), main cause Dyslipidemia in eighteen percent (18.2\%), Congenital Heart disease in eight percent $(8.3 \%)$ and by alcoholism there is four percent $(4.4 \%)$. The rate of mortality was $22 \%$ and most of the patients at the time of discharge were partially or fully dependent on other members or relatives for their daily and routine activities. [2][3][11] (See Figure 5).

Another set of data taken from the source i.e." National Institute of Cardiovascular Disease (NICVD), (a tertiary care Cardiac hospital, Karachi)" from their recent five years records, the descriptive data for this specific purpose is conducted from Patient's file records and thoroughly reviewed and the related details and disease information including symptoms and duration of disease recorded via a general predesigned questionnaire as well as a pro forma.

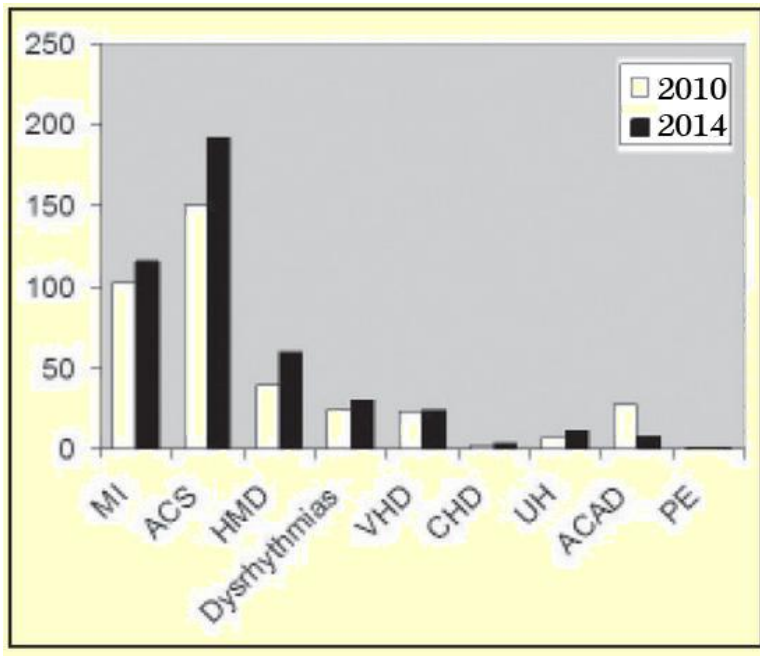

Figure 5: Cardio vascular disease pattern showing various risk factors at NICVD, Karachi, from recent five years

(2010-2014)[2]

www.jpma.org.pk/full_article_text.php?article_id=6240Intern ational journal of Pakistan Medical association. Last accessed on September 8, 2014

According to this second data set approximately seventy two $(72.5 \%)$ patients majority were male $(71 \%)$ were in fourth decade of their life. Hypertension and ACS (Acute Coronary Syndrome) was the major and the most common in patients approximately forty percent $(40 \%)$, the risk factor of hypertension disease followed by twenty seven (27.45) by MI (Myocardial Infarction) while ten percent $(10.4 \%)$ were identified Hypertension with Cardiac muscle disease. The rate of Mortality is $3.5 \%$ out of total 449 patients admitted in recent 5 years in that Hospital. The estimated ratio of mortality for the current year is approximately one percent (1.35\%). [9][14] (See Figure 5).

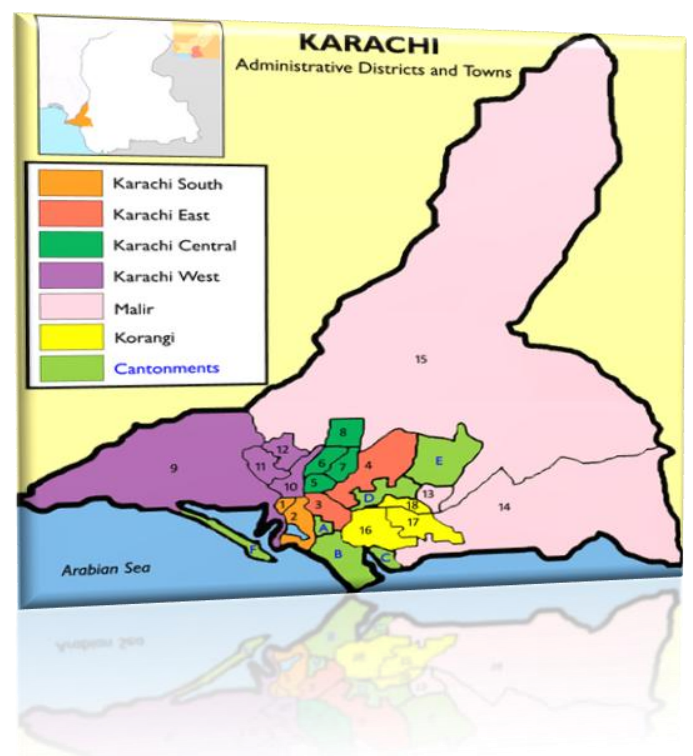

Figure 6: A Map of Karachi [1]

www.en.wikipedia.org/wiki/Karachi.Information about Karachi City. Retrieved 6 January 2014 
The conducted results and frequency explains the whole scenario of Hypertension admission risk of hospitalization in a very proper manner and the main concluding point from all the collected data sets of different hospitals of Karachi city that the Risk of Hypertension is high in low areas from economic and sociological perspectives, it also describe the modifiable multiple factors of hypertension disease risk of hospitalization admissions. Analysis of those data sets clearly shows that in recent years there is no as such change in the pattern of Hypertension disease is not changes as it can be changed in a significant manner and burden of this disease can also be decreased as compared to its past conventional pattern, it can be reduce able by awareness of people and also finest treatment for these risk factors can also reduce the rate of morbidity and mortality by Hypertension disease. [15]

\subsection{Standardized Ratio calculation}

The standardized ratio is used to estimate the level of specific population form the small regions of the particular areas. It is expressed as:

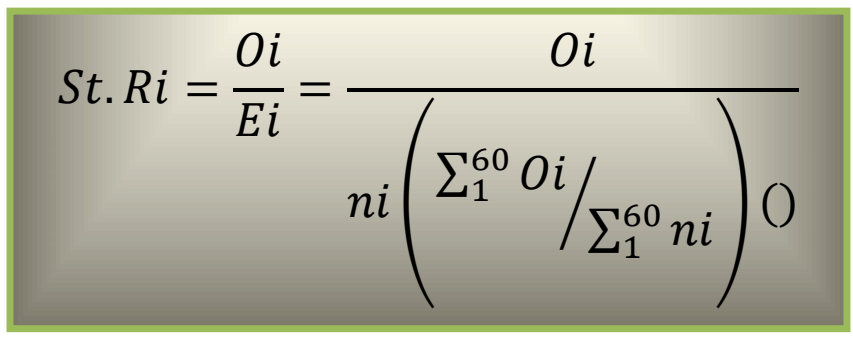

Notations:

$\mathrm{Oi}=$ denotes the "Observed counts of hospitalizations admission cases Hypertension disease, in any "i" district/sub district respectively".

$\mathrm{Ei}=$ "Estimated counts hospitalizations admission cases Hypertension disease in any "i" district/sub district respectively".

*Where $\mathrm{i}=$ any "i" district/sub district respectively.

$\mathrm{ni}=$ Represent the resident Population of each district/ subdistrict "i".

Therefore; the Standardized ratio determine the ratio of observed cases from data sets (observed counts) to the expected counts from the general outcomes of population in study area, Standardized ration ultimately give the occurrence of Hypertension disease in level in the specified area weather it will be high or low in that particular area.

Hence, the Hypertension disease data sets extracted from the various hospitalization admission cases but it does not contain local information of proper age factors, than we applied the same standardized ratio for age specific admission ratio in almost each region (sub- district) and it is useful to calculate the risk of hospitalization admission for Hypertension disease across the under study area of this research. [8]

The formula for Standardized ratio calculation further explain the computation that the no. of expected cases outcome from the multiplication of admission rate of Hypertension disease of general Inhabitants of particular area by resident of inhabitant of each of districts. This kind of Standardized ratio calculation is also called as Indirect Method of Standardized ratio Calculation.

It's also showed by standardized Ratio Formula that in some extent it will give the most appropriate levels like High or Low for the particular disease but proper and formal mapping cannot be possible with standardized Ratio Formula in an approximate way of computation for this kind of widely assumptive disease i.e. Hypertension.

\subsection{Bayesian Model-Based Disease Mapping}

To reduce the drawbacks of the presented Standardized Ratio Calculation, Bayesian Model instead of that standardized ratio is mostly adopted for various mapping of disease.

In this model we assumed a poison distribution for the hospitalization cases at each sub-district level and nos. of hospitalization admission in each Sub-region or sub district as "i", and " $\mathrm{i}$ " can be given by expected cases of hospitalization admission, and the Standardized ratio "St.Ri", so, the general form is formulated as:

$$
E(O i)=\mu i=E i X S t . R i
$$

\section{3- RESULTS}

The risk of hypertension can be variable in many regions In Karachi as well as there are many risk factors of hospitalization admission for Hypertension disease like family i.e. congenital heart muscle disease, age factors, smoking, physical disability, high intake of salt in diet, excessive usage of alcohol, condition of unconscious and permanent stress. Hypertension disease risk factor may also be varying according to region and its socioeconomic levels and environmental conditions. In this paper we have applied as much as possible cases of Hypertension disease from many sub-district and approximately thousands $(1,000)$ hospitalization admission cases recorded and tabulated for this purpose. The final outcomes and results showed that in low socioeconomic areas the highest percentage of hypertension disease as per calculation of standardized ratio calculation is $3.8 \%$ while in some other than the low socioeconomic regions the rate is $0.51 \%$ only.[7][10][16]

Table 1. Result showing rate of Hypertension Hospitalization Admission in Karachi

\begin{tabular}{|c|c|c|}
\hline $\begin{array}{c}\text { Risk of } \\
\text { Hypertension }\end{array}$ & Target Areas & $\begin{array}{c}\text { Rate of } \\
\text { Hypertension } \\
\text { hospitalization }\end{array}$ \\
\hline Variable & $\begin{array}{c}\text { low } \\
\text { socioeconomic } \\
\text { regions }\end{array}$ & $0.51 \%-3.8 \%$ \\
\hline
\end{tabular}

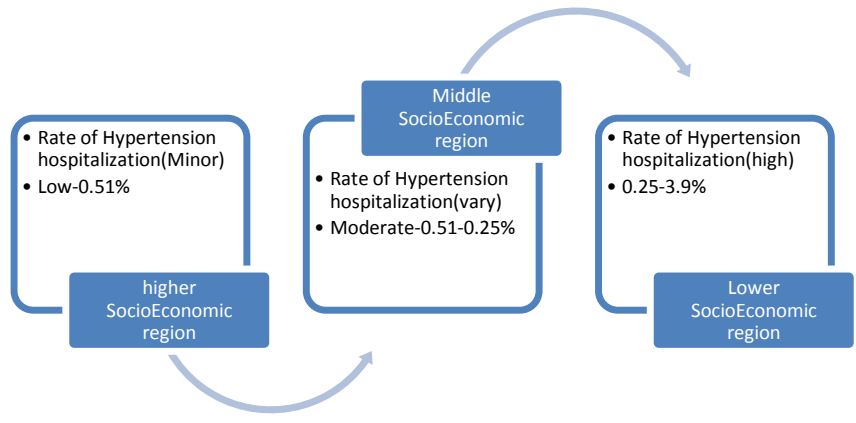

Figure 7: Variation in Rate of Hypertension Hospitalization Admission [5] 


\section{4- DISSCUSSION AND CONCLUSIONS}

In past years, many researchers have done their most of the work on the analysis of hypertension disease mapping and applied innovative techniques for this kind of widely influenced disease and its risk factors of the hospitalization admissions in many local areas of particular regions. We have applied Poisson distribution based on Bayesian model to map a finite pattern for the accurate ratio for hypertension disease admissions. [8][13]

In Karachi, Pakistan, the rate of Hypertension disease is increasing rapidly and the risk factors also increasing simultaneously with this disease and the requirement and treatment rate is very low comparatively for the hospitalization admission of hypertension disease that's why morbidity and mortality rate is also increasing in Karachi specifically in low socioeconomic inhabitant areas.[10]

Bayesian model is used for Hypertension disease's hospitalization admission mapping for small population regions and the statistics form this model showed that the smooth and proper relative risk factor for Hypertension disease across a geographical area of Karachi shown in Fig.2, 3 and 4.

The recent studies, researchers only describe the Hypertension disease risk factor on large areas and over some limited factors like prevention, modification and quality of lifestyle of specific inhabitant of that area also demonstrate that the population affected by hypertension disease is largely depend upon some general factors. In this study, we examine and strived to explore the spatial variation of hospital admission for hypertension disease over a wide scope of heterogeneity of relative risk factors in many sub district through Karachi, Pakistan in past fifteen 15 years.[7]

\section{5- FUTURE RESEARCH SCOPE}

This work can be further modified as on big data for multiple cities of Pakistan. The concept of this work is based upon a specific city i.e. Karachi and the result shows some common outcomes for low socio economic areas of Karachi. So, The Future idea could be extended in the same manner on multiple targeted cities with variable data. Big Data Strategy would be implying on this vast field of study.

\section{6- REFRENCES}

[1] Information about Karachi City. www.en.wikipedia.org/wiki/Karachi. Retrieved 6 January 2014

[2] International journal of Pakistan Medical association. www.jpma.org.pk/full_article_text.php?article_id=6240I Last accessed on September 8, 2014

[3] An official website of Pakistan stroke society. Last accessed on April 5, 2014. Www.pakstroke.com.

[4] World Health Organization (WHO). The Atlas of Heart Disease and Stroke. http://www.who.int/cardiovascular_diseases/resources/atl as/en/, Retrieved 6 January 2014.

[5] College of Physicians and Surgeons Pakistan. http://www.cpsp.edu.pk/index.php?code $=Z$ ZGVwYXJ0b WVudHN8YXNkLnBocHxibHMucGhwfDB8MA== Last accessed on April 5, 2014.

[6] A. Defontaine, A. Hern_andez, and G. Carrault (3504/2005,13/06/2005). "Multi-Formalism Modelling of Cardiac Tissue," HAL author manuscript, Lecture notes in computer science; 394-403.

[7] Pakistan Medical Research Council: National Health Survey of Pakistan 1990-94. Islamabad, Pakistan Pakistan Medical Research Council, Network Publication Service.

[8] Saleheen D, Frossard P. CAD(2004) risk factors and acute myocardial infarction in Pakistan. Acta Cardiol;59(4):417-24.

[9] Allen CMC, Lueck CJ. Carebrovascular diseases, Davidsons principles and practice of Medicine (19th edition), Haslett C., Chilvers ER., Boon NA., Colledge NR, Hunter JAA, (Eds), (2002;1159-68).Churchill living stone, London, U.K.

[10] Hansell, A.L.; Blangiardo, M.; Fortunato, L.; Floud, S.; de Hoogh, K.; Fecht, D.; Ghosh, R.E.; Laszlo, H.E.; Pearson, C.; Beale, L.; et al (BMJ 2013) Aircraft noise and cardiovascular disease near Heathrow airport in London: Small area study. 347, doi:10.1136/bmj.f5432.

[11] Akhter W. Stroke: (1999).The Common modifiable risk factors and inpatients outcome. Dissertation College Physicians Surg Pak (CPSP). Med / 867/

[12] Ali L, Jameel H, Shah MA. Risk factors in stroke. J Coll Physicians Surg Pak 1997;7:7-10.

[13] Islam, M.R.; Khan, I.; Attia, J.; Hassan, S.M.N.; McEvoy, M.; D'Este, C.; Azim, S.; Akhter, A.; Akter, S.; Shahidullah, S.M.; et al.(2012,). Association between hypertension and chronic arsenic exposure in drinking water: A cross-sectional study in Bangladesh. Int. J. Environ. Res. Public Health ,9, 4522-4536.

[14] Lewington S, Clarke R, Qizilbash N.( 2002). Agespecific relevance of usual blood pressure to vascular mortality: A meta-analysis of individual data for one million adults in 61 prospective studies. Lancent ;360:1903-2191.

[15] Rafique BA, Yousuf M, Iqbal J, Khan MM. (2000,2002) Frequency of known risk factors for stroke in poor patients admitted to Lahore General Hospital inPak J Med Sci;18:280-3

[16] Colditz GA, Bonita R, Stampfer MJ, Willett WC, Rosner $\mathrm{B}$, Speizer FE, et al. Cigarette smoking and risk of stroke in middle aged women. N Engl J Med 1988;318:937-41. 